\title{
Caracterización de las cenizas volantes de una planta termoeléctrica para su posible uso como aditivo en la fabricación de cemento
}

\section{Characterization of flying ashes of a thermoelectric plant for its possible use as an additive in the manufacture of cement}

\author{
William Alexander Bautista-Ruiz' \\ Mercedes Díaz-Lagos ${ }^{2}$ \\ Segundo Agustín Martínez-Ovalle 3
}

Recibido: marzo 06 de 2017

Aceptado: junio 29 de 2017

\section{Resumen}

En este trabajo se presentan los resultados de la caracterización de Cenizas Volantes, CV, producto de la combustión del carbón, las cuales fueron producidas en la estación termoeléctrica Termopaipa IV, de la región de Boyacá, Colombia. Las técnicas empleadas para la caracterización fueron: Fluorescencia de Rayos X , Difracción de Rayos X, Microscopía Óptica (Sección Delgada Pulida), Microscopía Electrónica de Barrido y Espectroscopía de Fotoelectrones de rayos $X$. Se observa que las cenizas presentan principalmente contenido de compuestos de aluminio y silicio, además, fases de hierro en menor escala como son Hematita y Limonita. La morfología revela inquemados de carbón y esferas de diferente tamaño y composición. A partir de las características encontradas en este estudio como son: composición química, mineralogía y características superficiales, se determina que las CV son un material puzolánico artificial con carácter ácido lo cual influye sobre la reactividad de las cenizas que se basa en el aspecto químico de la fijación de la cal y cumplen con los requerimientos para su utilización en la industria cementera.

Palabras clave: ceniza volante, propiedades fisicoquímicas y morfológicas, reactividad superficial, actividad puzolana, industria cementera.

\begin{abstract}
The results of the characterization of 'Flying Ashes', FA, a product of coal burning are shown in this paper. The 'flying ashes' samples were produced in the thermal station Termopaipa IV, in the State of Boyacá, Colombia. The techniques used for the characterization were: X-Ray Fluorescence, X-ray diffraction, Optical Microscopy (Polished Thin Section) and Electronic Microscopy. It is noticeable that the ashes are made up of aluminum and silicon compounds. Besides, iron phases in low content such as hematite and limonite were also found. Additionally, the morphology reveals presence of coke unburned coal and spheres of different size and composition. According to the features found in this study, such as: chemical and mineralogical composition, and surface characteristics have determined that the FA is an artificial pozzolanic material with acidic properties, which influences the reactivity of the ashes that is based on the chemical aspect of the lime fixing and fulfil the requirements in a high degree according to the standard for use in the cement industry.
\end{abstract}

Keywords: fly ash, physicochemical and morphological properties, surface reactivity, puzzolanic activity, cement industry.

1 Físico, Universidad Pedagógica y Tecnológica de Colombia, Tunja, Colombia. E-mail: william.bautista@uptc.edu.co

2 Física, Doctora en Física de la Materia Condensada y Nanotecnología, Universidad Pedagógica y Tecnológica de Colombia, Sogamoso. E-mail: mercedes.diaz@uptc.edu.co

3 Licenciado en Física y Matemáticas, Doctor en Bioingeniería y Física Médica, Universidad Pedagógica y Tecnológica de Colombia, Tunja, Colombia.E-mail: s.agustin.martinez@uptc.edu.co 


\section{Introducción}

En la actualidad el carbón juega un papel importante en la producción de energía eléctrica a nivel mundial. En Colombia, la principal fuente de producción de energía eléctrica son las estaciones termoeléctricas gracias a la gran reserva de carbón con que cuenta el país; en éstas, la utilización del carbón como materia prima, repercute de manera significativa a nivel ambiental ya que se generan residuos contaminantes como las Cenizas Volantes, CV. Las CV poseen diferencias en su composición química, su mineralogía y granulometría, dependiendo de ciertas variables, que generalmente se engloban en el tipo de carbón que se quema y del proceso de combustión como: temperatura, tiempo de residencia del carbón, tipo y geometría de la caldera donde tiene lugar la combustión, configuración de fuegos y marcha de la combustión (Vassielev, \& Vassileva, 2005; Jegadeesan, Souhail, Abed, \& Pinto, 2008; Fly Ash India Pvt. Ltd, 2014; Santaella-Valencia, 2001; Reyes-Caballero, Fernández-Morales, \& Duarte, 2016; Martínez-Bernal, 2013).

En la región de Boyacá la importancia de este residuo o subproducto es significativa, ya que en una sola planta termoeléctrica aproximadamente se producen 200 toneladas (t) de CV por cada $1500 \mathrm{t}$ de carbón quemado al día, es decir $13 \%$ de la materia prima se convierte en desecho (Reyes-CabaIlero, Martínez-Ovalle, \& Moreno-Gutiérrez, 2015); esto significa que una gran cantidad de material de desecho se produce anualmente y que en la planta termoeléctrica ya no tiene ningún uso.

A nivel mundial se investigan soluciones enfocadas a la utilización de este material como materia prima para múltiples aplicaciones como la fabricación de cemento, ya que posee componentes puzolánicos como Silica (SiO2) y Alúmina (Al2O3) (Wang, \& Wu, 2006). Adicionalmente las CV poseen óxidos de hierro (Fe2O3), el cual se puede recuperar siempre y cuando esté presente en determinado porcentaje para que sea viable para las industrias siderúrgicas; en muchos casos puede poseer elementos trazas, como As, Se e inclusive $\mathrm{Pb}$ originarios del carbón utilizado (Gómez-Rojas, Díaz-Lagos, Blandón-Montes, \& Martínez-Ovalle, 2016). También contiene carbón inquemado que resulta útil en la fabricación de ladrillos ya que proporciona valor energético en el proceso de sinterización (López, \& González, 1995). Otros usos de las CV del carbón es en la conversión en zeolitas (Querol et al., 2002; Murayama, Yamamoto, \& Shibatia, 2002; Querol, Alastuey, Turiel, \& López, 1995); así como para propósitos de inmovilización como por ejemplo en el caso de residuos radiactivos (Lieberman et al., 2015; Martínez-Ovalle, Reyes-Caballero, \& González-Puin, 2013; Vasileva, Anshits, Sharonova, Burdin, \& Anshits, 2005).

Basándose en la composición química, que fundamentalmente depende de la naturaleza del carbón utilizado y la mina donde ha sido extraído (Fly Ash India Pvt. Ltd, 2014; Santaella-Valencia, 2001), las CV pueden clasificarse según la norma ASTM C618 (2005) y su equivalente para Colombia, la norma NTC-3493 (1993) en dos tipos principales: F o C, cuya diferencia fundamental está en el contenido de Ca. Las cenizas tipo F son producidas generalmente por la combustión de carbones bituminosos o antracíticos y su contenido mínimo de $\mathrm{SiO} 2$ + $\mathrm{Al} 2 \mathrm{O} 3$ + $\mathrm{Fe} 2 \mathrm{O} 3$ es del 70\%, usualmente menos del $10 \%$ de $\mathrm{CaO}$ (propiedades puzolánicas y valor cementante nulo) y con porcentaje de inquemados entre el $2 \%$ y el $10 \%$ (pérdidas por ignición, que depende del origen y tipo de carbón, de la finura del carbón y la temperatura de la caldera). En cementos, para que no se afecte la resistencia y estabilidad, los porcentajes por pérdida de ignición no deben ser mayores al 12\% (Valderrama, Agredo, \& de Gutiérrez, 2011). Las CV tipo C producidas por la combustión de carbones sub-bituminoso o lignito poseen un contenido mínimo de $\mathrm{SiO} 2+$ $\mathrm{Al} 2 \mathrm{O} 3+\mathrm{Fe} 2 \mathrm{O} 3$ del $50 \%$ y contienen desde un 10\% hasta un $40 \%$ de $\mathrm{CaO}$ (propiedades puzolánicas y cementantes), con pérdidas por ignición hasta del $6 \%$.

La actividad puzolánica de las CV depende principalmente del tipo y cantidad de fase vítrea, de 
la finura de sus componentes mineralógicos, de la morfología de sus partículas y del nivel de inquemados o materia orgánica presente, expresada como la pérdida al fuego de éstas (Mehta, 1989). Se han reportado resultados diversos acerca del efecto del carbón inquemado en las propiedades físicas, químicas y mecánicas de concretos adicionados con CV (Yua et al., 2012; Jensen, \& Rasmussen, 2008; Ahmaruzzaman, 2010). En el cemento se mencionan mejoras en la fuerza, cualidades como barreras contra la humedad y las características de resistencia a la abrasión (Nassara, Soroushian, \& Ghebrabc, 2013). Estas mejoras son provocadas por la reacción puzolánica de la CV con los hidratos de cemento que cambia favorablemente la microestructura y la zona de transición de interfase en el hormigón, concluyéndose que el uso de CV en el hormigón puede economizar significativamente la construcción de pavimentos de hormigón y mejorar la vida útil de la infraestructura vial (Nassara et al., 2013). Otras de las mejoras de adicionar CV son: incremento en la trabajabilidad y disminución de la permeabilidad (Lorenzo-García, 1993); reducción del calor de hidratación, aumento de la resistencia mecánica a largo plazo (Raghu, Eskandari, \& Reddy, 2009; Naik, Singh, \& Ramme, 1998); y buen desempeño frente a ambientes agresivos (Ahmaruzzaman, 2010; Gopalan, 1996; Sahmaran, Yaman, \& Tokyay, 2009; Alhozaimy, Soroushian, \& Mirza, 1996; Ampadu, \& Torii, 2002; ASTM, 2005). Un uso más reciente ha sido el de atenuar ondas electromagnéticas, debido a que es posible mejorar sus potencialidades mediante la introducción de poliestireno expandido y CV (Baoyi, Yuping, \& Shunhua, 2012).

En Colombia se han utilizado las CV en la industria cementera y en otras aplicaciones de interés científico y tecnológico (Valderrama et al., 2011; Aperador, Ruiz, \& Delgado, 2014; Pedraza, Pineda, \& Gutiérrez, 2015). En el caso de la industria cementera, la actividad puzolánica depende de la composición química, mineralógica y la distribución granulométrica, la estructura y estado de la superficie. Es por esto que se hace necesario conocer sus propiedades para entender la reactivi- dad de las CV y su valor puzolánico, puesto que esas propiedades y no la procedencia del material, determinan la influencia de la adición sobre el comportamiento del cemento u hormigón que lo contiene. En este trabajo se muestran entonces los resultados de la caracterización composicional, estructural, morfológica de las CV producidas en la central termoeléctrica de Boyacá, al igual que propiedades químicas de la superficie, poniendo en evidencia que este material cumple con los requerimientos para su utilización.

\section{Materiales y métodos}

En este trabajo se analizaron CV producto de la combustión del carbón suministradas por la Compañía Eléctrica de Sochagota SA, Termopaipa IV, ubicada en el km 5 carretera Paipa a Tunja, en Boyacá, Colombia. Las muestras se sometieron a un proceso de homogenización, con la finalidad de obtener una muestra representativa.

La composición elemental se determinó usando un espectrómetro de Fluorescencia de Rayos $X$ de energía dispersa PANalytical, modelo Axios Petro de 4 kW, con un detector de Xenón (Xe) de centelleo de flujo y ánodo de Rodio (Rh). Para identificar las fases cristalinas y mineralógicas presentes en las CV, se utilizó un difractómetro de rayos $X$ marca PANalytical. Las medidas se tomaron en un rango de entre $2 \theta=10^{\circ}$ y $2 \theta=70^{\circ}$. Para obtener información de la morfología de las CV y estimar la composición de la muestra se realizó el análisis petrográfico por microscopía óptica, utilizando la técnica de sección delgada pulida (SDP) que permite la observación de minerales e inquemados presentes en la superficie de las CV. Se utilizó un microscopio petrográfico Leica DM 4500P de Luz polarizada.

La caracterización morfológica y de composición química se llevó a cabo en un microscopio electrónico de barrido, modelo Nova Nano SEM 230, en el Instituto de Ciencia de Materiales de Madrid, CSIC-ICMM, donde también se realizaron las medidas de Espectroscopía de Fotoelectrones de Rayos 
$X(X P S)$, que es una técnica semi-cuantitativa para el estudio de la composición y estructura electrónica de la muestra a nivel superficial. Las medidas se realizaron en una cámara en ultra-alto vacío, donde el ángulo entre el analizador hemisférico (SPECS-PHOIBOS100) y el plano de la superficie se mantuvo a $60^{\circ}$ y la radiación de Rayos $X$ fue la línea Ka del magnesio (Mg) (1253.6 eV). La escala de la energía de enlace (BE) fue calibrada con respecto al carbono (C) $1 \mathrm{~s}$ para $285 \mathrm{eV}$.

\section{Resultados y discusión}

3.1 Análisis composicional por fluorescencia de rayos $X$

La composición química de las cenizas volantes (CV) se presenta en la Tabla 1. Esta composición está dada en forma de óxido y expresada en porcentaje en peso (comp. \%Wt).

\begin{tabular}{llllll}
\hline Compuesto & Comp.\% Wt & Compuesto & Comp.\% Wt & Compuesto & Comp.\% Wt \\
\hline $\mathrm{SiO}_{2}$ & 64.87 & $\mathrm{TiO}_{2}$ & 1.20 & $\mathbf{S r O}$ & 0.11 \\
$\mathrm{Al}_{2} \mathrm{O}_{3}$ & 24.62 & $\mathbf{P}_{\mathbf{2}} \mathbf{O}_{5}$ & 0.66 & $\mathbf{B a O}$ & 0.10 \\
$\mathrm{Fe}_{2} \mathrm{O}_{3}$ & 4.51 & $\mathbf{N a}_{\mathbf{2}} \mathbf{O}$ & 0.52 & $\mathbf{C u O}$ & 0.09 \\
$\mathrm{~K}_{2} \mathrm{O}$ & 1.36 & $\mathbf{M g O}$ & 0.40 & $\mathbf{V}_{\mathbf{2}} \mathbf{O}_{\mathbf{5}}$ & 0.05 \\
$\mathrm{CaO}$ & 1.33 & $\mathbf{S O}_{3}$ & 0.12 & $\mathbf{Z r}_{\mathbf{2}} \mathbf{O}_{\mathbf{2}}$ & 0.03 \\
\hline
\end{tabular}

Tabla 1. Composición elemental de las cenizas volantes en orden creciente de concentración de especies, obtenidas mediante FRX.

Según los resultados de la tabla 1 , la composición química de la CV utilizada en el presente estudio posee concentración de elementos mayoritarios de óxido de Silicio (SiO2), óxido de Aluminio (Al2O3) y óxido de hierro, (Fe2O3). Existen contenidos de otros metales, pero en fracciones menores. Los resultados indican que las CV son en su mayoría compuestos aluminosilicatos.

La concentración de elementos mayoritarios es de gran importancia por su influencia en la potencial aplicación de las CV, y la concentración de algunos elementos traza pueden tener importancia en cuanto al impacto ambiental en su utilización. Los análisis revelan que las CV de la planta termoeléctrica analizada están constituidas por cenizas del Tipo $\mathrm{F}$, debido a que tiene $\geq 70 \%$ de $\mathrm{SiO} 2+\mathrm{Al} 2 \mathrm{O} 3+-$ Fe2O3, con una relación Si/Al del (2.63) y bajos contenidos de otros elementos como: $\mathrm{K}, \mathrm{Ca}, \mathrm{Ti}, \mathrm{P}$, $\mathrm{Na}, \mathrm{Mg}, \mathrm{S}, \mathrm{Sr}, \mathrm{Ba}, \mathrm{Cu}, \mathrm{V}$ y Zr. Es de esperar esta clase de CV, ya que el carbón que llega a la planta es del tipo sub-bituminoso y las pérdidas por ignición son del $8.14 \%$, valor aceptado para este tipo de cenizas, pues la norma permite hasta $12 \%$ de pérdida por ignición (NTC 3493,1993). En este sentido, las cenizas objeto de estudio cumplen los requerimientos para ser utilizadas en la fabricación de cemento.

Según Fauzi y colaboradores (2016), la importancia de las cenizas tipo $\mathrm{F}$ radica en que al poseer gran cantidad de Al y Si se constituyen en cenizas que proporcionan aluminosilicatos amorfos, con la ventaja de reaccionar mejor con los hidróxidos de calcio $(\mathrm{Ca}(\mathrm{OH}) 2)$, el cual es liberado en el proceso de hidratación del cemento. Cuando hay un exceso de $\mathrm{CaO}$ (compuesto básico), los compuestos $\mathrm{Al} 2 \mathrm{O} 3$ y $\mathrm{Fe} 2 \mathrm{O} 3$ se comportan como ácidos al igual que el SiO2 (Goma, 1975). En este caso las CV tienen una naturaleza ácida, pues si bien la cantidad de $\mathrm{CaO}$ no es superior a los compuestos de Fe o Al, la presencia de $\mathrm{SiO} 2$ es superior a cualquier otro compuesto presente. Una ventaja importante de la baja presencia de $\mathrm{CaO}$ en las cenizas tipo $\mathrm{F}$ es que son más eficientes cuando se trata de suprimir 
la expansión debida a la reacción de sílice alcalina (Hemalatha, \& Ramaswamy, 2017).

\subsection{Caracterización mineralógica por difracción de rayos $X$}

El espectro DRX de la CV se muestra en la figura 1. Se presenta tanto la fase cristalina y la fase amorfa, en donde esta última se observa en la curvatura de la base del patrón comprendida aproximadamente entre $2 \theta=10^{\circ}$ y $40^{\circ}$, las fases cristalinas encontradas son: Mullita (M), Cuarzo (Q) y Hematita (H).

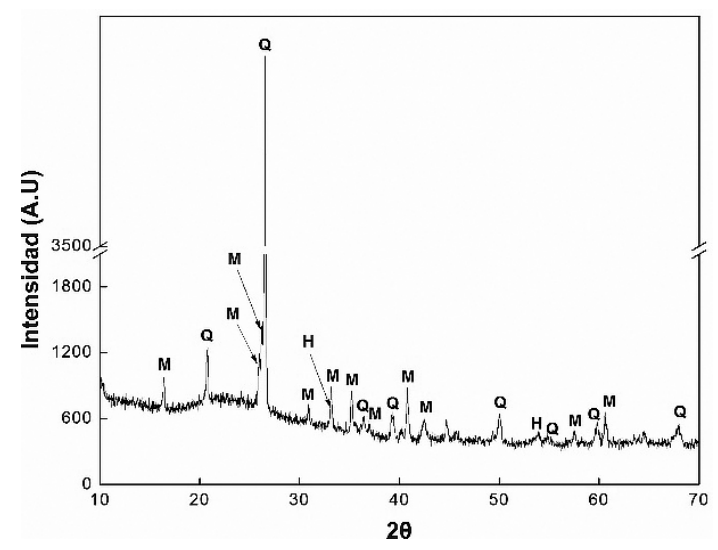

Figura 1. Patrón de difracción de las cenizas volantes obtenida con radiación Cu-Ka $(\lambda=1.54060 \AA$ ) con velocidad de escaneo $0,020^{\circ} / 2 \mathrm{~s}$.

A partir del análisis cualitativo se identificaron las fases estructurales presentes, que corresponden a dos fases mayoritarias: Mullita (Al4.80 09.60 Si1.20), Cuarzo (SiO2), y una tercera fase estructural en menor proporción asociada a Hematita (Fe2O3); fases que son esencialmente pasivas que posibilitan la formación de hidratos de cemento (Fauzi et al., 2016). Algunas de estas fases se evidencian al ser observadas mediante microscopía óptica, las cuales se describen más adelante. Cada una de estas fases se compararon con fichas preexistentes en el Powder Difrraction File, PDF, del International Centre for Difraction Data, ICSD: 066263; 079634 y 015854, respectivamente.
De las tres fases que presentan las cenizas, se observa que todas contienen Al, Si y Fe lo cual es coherente con los resultados obtenidos del análisis elemental, donde se encontró que los óxidos mayoritarios en orden descendente son: Silicio (SiO2); Aluminio (Al2O3) y Hematita (FeO3) fases que corresponden con las reportadas por otros autores (Van der Merwe, Mathebula, \& Prinsloo, 2014; Wu et al., 2011; Ha et al., 2005). La cantidad restante de elementos presentes está contenida en la parte amorfa de este material.

\subsection{Microscopía óptica: sección delgada pulida}

La microscopía óptica proporciona información superficial morfológica y composicional de las CV. Los compuestos más comunes que se encuentran son: carbón, vidrios y minerales y la forma física es de partículas de diferentes tamaños llamadas: microesferas vítreas, cenosferas, plerosferas y en ocasiones magnetosferas (Vassilev, \& Vassileva, 2005; Zyryanov, Petrov, \& Matvienko, 2011; Szumiata et al., 2014). Se identificaron carbones inquemados de naturaleza isotrópica, anisotrópica así como inertinita y algunos tipos de vidrio como son: básicos, medio ácidos, ácidos masivos, vesiculares y ferriesferas. Las CV de bajo contenido de calcio presentan un porcentaje alto de sílice y alúmina, su morfología corresponde a partículas esféricas vítreas, aluminosilicatos, que en ocasiones contienen hierro $(\mathrm{Fe})$ y álcalis. Presentan una proporción de partículas de carbón sin quemar o parcialmente quemadas muy pequeñas, aproximadamente $<2 \%$ en relación con las de alto contenido en $\mathrm{Ca}$. Sin embargo, las CV que poseen alto contenido de calcio (Ca) con frecuencia presentan un porcentaje de inquemados que oscila entre el $2 \%$ y el $10 \%$. Este carbón inquemado puede estar encerrado en las partículas esféricas vítreas, pero en mayor proporción aparece en las partículas que poseen forma celular o vesicular de tamaño superior a los $45 \mu \mathrm{m}$ con apariencia porosa y una gran área superficial (Vassilev, \& Vassileva, 2005).

En la figura 2 se muestra la imagen de las CV en la que aparece el carbón inquemado coque que se 
observa en las zonas claras, de forma irregular y de apariencia porosa. También se encuentra cuarzo, el cual es obtenido por otras técnicas como DRX; además metales y óxidos de hierro y se identifican algunos minerales metálicos de forma esférica que se muestra en la imagen de la figura 3. Es de resaltar que el carbón inquemado remanente en las CV tiene gran importancia. Según Pedraza y colaboradores (2015), las propiedades mecánicas y el aumento en el tiempo de curado en los morteros de cemento portland mejoran con la adición de CV provenientes de la misma región con determinadas cantidades de carbón inquemado.

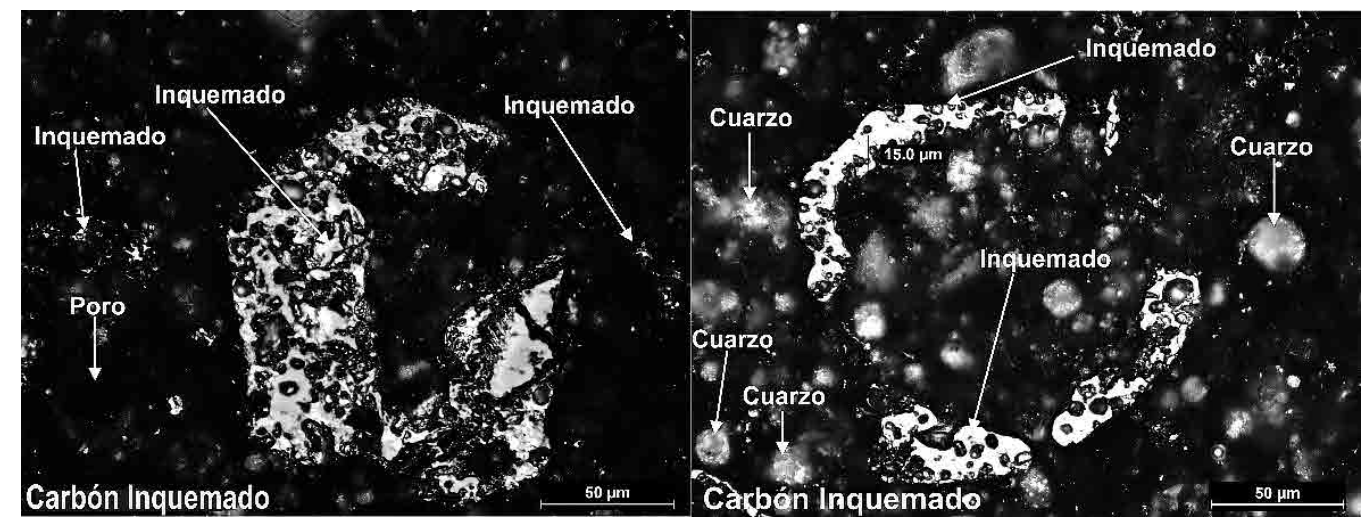

Figura 2. Micrografía de sección delgada pulida vista con luz reflejada de la muestra de CV.
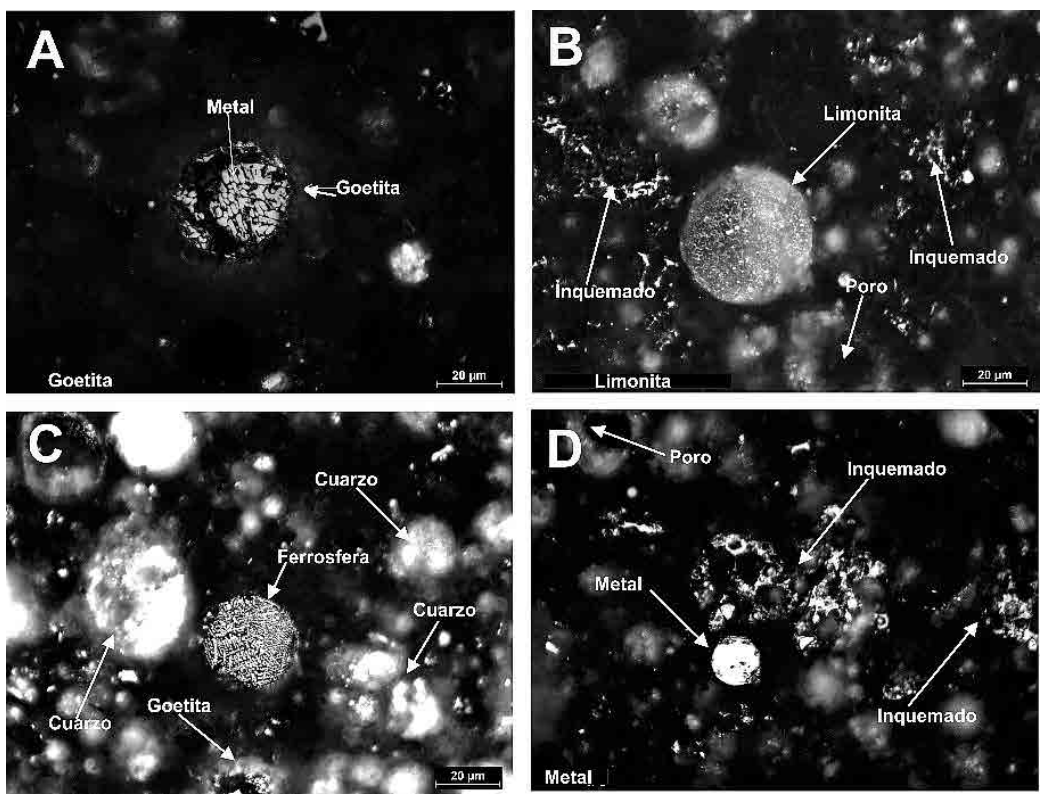

Figura 3. Micrografía óptica de sección delgada pulida, vista con luz reflejada y microscopio petrográfico.

En la figura 3(A) se observa la presencia de goetita, cuyo origen está asociado a un óxihidróxido de hierro (III). Una característica particular de este tipo de óxido es su color rojizo indicando la oxida- ción de este mineral. En la figura 3 (B) se evidencia la presencia de limonita y carbón inquemado y en la figura $3(C)$ se muestra nuevamente la goetita, 
cuarzo y lo que puede ser una ferrosfera, según lo reportado por Zyryanov y colaboradores (2011).

En la figura 3 (D) se identifican partículas metálicas esféricas con morfología lisa y brillante. También se identifican partículas metálicas, con forma esférica y texturizada, las cuales poseen una gran área superficial y por tanto una mayor reactividad que las lisas y las demás estructuras que se observan.

En resumen, del estudio microscópico se observó que muchos minerales y fases en las $C V$ tienen un tamaño de grano $<5-10 \mu \mathrm{m}$ (normalmente $\sim 10 \%$ -
$40 \%)$, en particular correspondiente a minerales. EI reconocimiento de minerales de baja reflectancia o aquellos íntimamente asociados con el carbón y vidrio no fue posible determinarlos mediante esta técnica.

\subsection{Análisis morfológico y elemental mediante FE-} SEM

En la figura 4 se presentan la morfología y composición de las CV mediante microscopía electrónica de barrido.
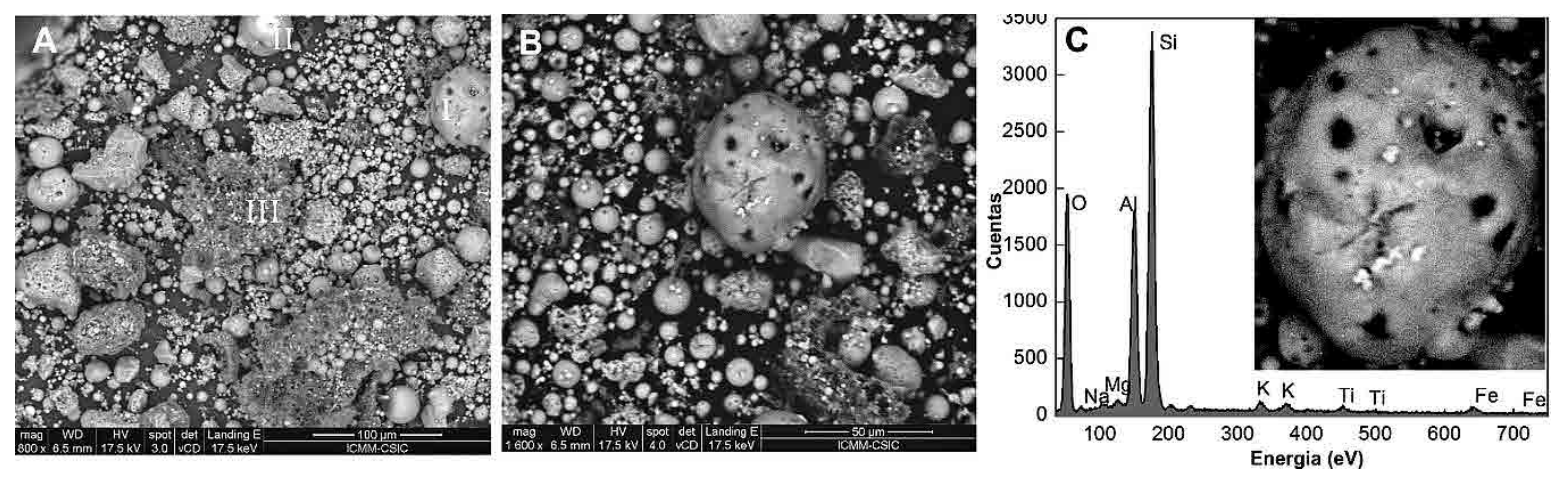

Figura 4. Imágenes SEM (A y B) imagen panorámica de CV donde se aprecia en detalle los diferentes componentes y su morfología, (C) composición química de una partícula.

Se observan partículas con simetría esférica de diferentes tamaños (I, II), así como partículas de forma irregular y con apariencia porosa (III). Un ejemplo de los detalles morfológicos y composicionales para una de estas partículas señalada como I, se observa en la figura 4 (B) tomada a $1600 X$ y su espectro composicional correspondiente (C) indica que sus componentes mayoritarios son aluminio y silicio. Cuanto más pesado el elemento, mayor cantidad de electrones son retrodispersados y generan zonas más brillantes en la imagen y viceversa, un ejemplo de ello se presenta en la partícula indicada con I. En las imágenes se observa gran contenido de cenosferas, las cuales son partículas esféricas que corresponden a aluminosilicatos, además, componentes de naturaleza irregular y estructura porosa que se asocian a inquemados de carbón (zona III) que por sus características morfo- lógicas se intuye el mecanismo de formación de las cenosferas y demás componentes asociados al proceso de combustión del carbón.

La forma de la partícula de CV en la figura 4 (C) es una esfera semiamorfa cuya composición química corresponde a $\mathrm{Si}, \mathrm{Al}$ y $\mathrm{O}$, tal como se indica en el espectro y que corresponden a los picos con más intensidad o número de cuentas. En coherencia con lo reportado por Fauzi y colaboradores (2016), se puede denominar a este tipo de esferas que presentan estas particularidades, esfera de aluminosilicato amorfo. Además se observan otros picos cuya intensidad es relativamente débil y que corresponden a Mg, $\mathrm{K}, \mathrm{Na}$, Fe y $\mathrm{Ti}$, estas intensidades indican las menores concentraciones de las partículas en estos elementos. El porcentaje de contenido químico mencionado se puede deter- 
minar mediante otros análisis como es el caso de la técnica de FRX (Fauzi et al., 2016).

\subsection{Análisis elemental superficial con XPS}

El análisis superficial de las CV mediante XPS, se presenta en la figura 5 . En A) se muestra que las
CV contienen principalmente $\mathrm{O}$ y $\mathrm{C}$ con presencia minoritaria de $\mathrm{Si}, \mathrm{Al}, \mathrm{S}, \mathrm{P}, \mathrm{Ca}, \mathrm{N}$ y Fe; resultados similares para muestras de CV han sido reportados por diferentes autores (Van der Merwe, et al., 2014; Chujiang et al., 2003).
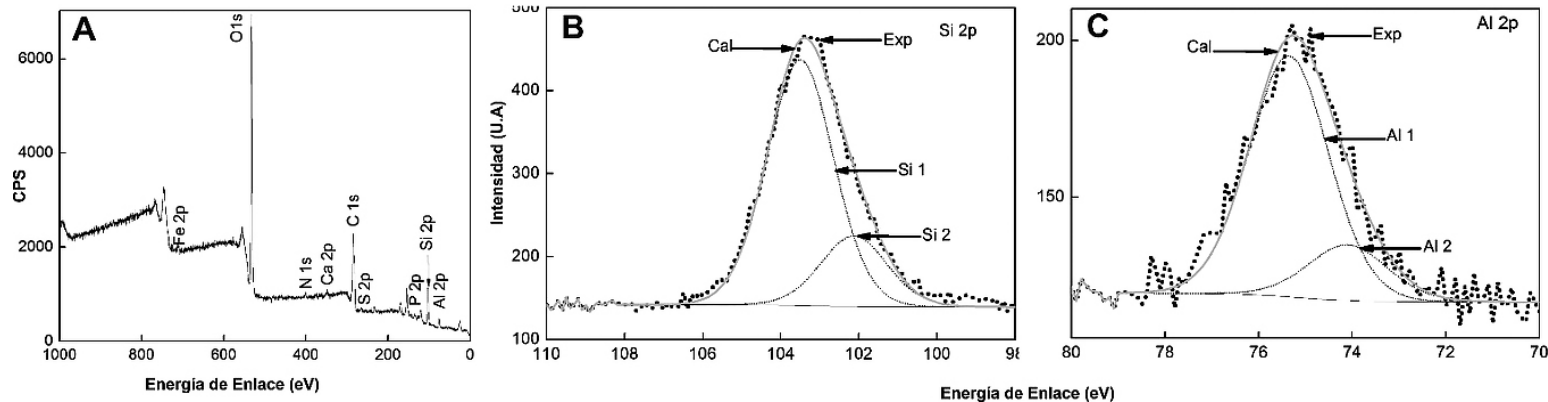

Figura 5. Espectro XPS de la superficie de la muestra de CV niveles Si $2 p$ (B) y Al 2p (C) de la superficie de CV.

La figura 5 ( $\mathrm{B}$ y C) muestra los espectros XPS de los niveles Si $2 p$ y Al $2 p$ presentes en la superficie de las CV, cuyos picos se sitúan en 103.7 eV y 75.7 $\mathrm{eV}$, respectivamente. Estos resultados permiten inferir que los posibles estados químicos para el oxígeno O con nivel 1s; Si con nivel 2p; Al con nivel $2 p$, pueden ser $\mathrm{CaSO} 4$, silicato y haluro/óxido, en coherencia con lo reportado por Van der Merwe y colaboradores (2014). Además, el trabajo de Nir y otros (2015), establece que la alta concentración de Si y Al, puede dar origen a formación de grupos aluminatos y silicatos, que a su vez son responsables de la carga negativa de la superficie, que en este caso también corresponde con lo encontrado. Las convenciones (Exp) hacen referencia a los datos experimentales; la notación $\mathrm{Si} 1, \mathrm{Si} 2$ y Al 1 Al 2 hace referencia a la deconvolución de cada nivel y la convención (Cal) corresponde al nivel calculado resultante luego de hacer el ajuste gaussiano para cada nivel.

\section{Conclusiones}

Análisis como FRX y DRX revelan que las Cenizas Volantes, CV, tipo $\mathrm{F}$ analizadas en este trabajo tienen un gran potencial para ser utilizadas como aditivo para la fabricación de cemento; principalmente por la presencia de aluminosilicatos amorfos propios de este tipo de ceniza los cuales reaccionan eficazmente con los hidróxidos de calcio $(\mathrm{Ca}(\mathrm{OH}) 2)$. Se encontraron las fases de Mullita (Al4.80 09.60 Si1.20), Cuarzo (SiO2), y Hematita (Fe2O3) que se constituyen en fases pasivas que posibilitan la formación de hidratos de cemento. Además, los resultados indican un bajo contenido de $\mathrm{CaO}$ lo cual da una ventaja importante a este tipo de cenizas, pues aumenta la eficiencia de suprimir la expansión debida a la reacción de la sílice alcalina.

El análisis morfológico permitió observar particularidades presentes en las CV, como el caso del carbón inquemado remanente que, según lo reportado en la literatura, adiciona ventajas mecánicas y tiempo de curado al cemento portland cuando son mezcladas con el. Bajo estas consideraciones, este material es un muy buen aditivo en la fabricación del cemento.

En general, los resultados indican que las CV tomadas de la combustión del carbón suministradas por la Compañía Eléctrica de Sochagota S.A. E.S.P 
- Termopaipa IV, ubicada en la región de Boyacá, Colombia, cumplen con los requerimientos para utilizarlas en la industria cementera. Las CV de esta central termoeléctrica, debido a su cantidad de inquemados, constituyen la materia prima para utilizarse en la fabricación de ladrillos ya que pueden aportar energía en la fase de sinterización. Si bien los contenidos de aluminio y hierro son bajos (Al2O3 24.62\%) (Fe2O3 4.51\%), en comparación con la cantidad de aluminio y hierro necesaria presente en las CV para que sea aceptada por las plantas de alúmina y acerías para la extracción de estos, se puede pensar en un proceso de beneficio con el cual puedan extraerse estos tipos de óxidos.

En la investigación se encontró que el uso de las CV en la región analizada es escaso; esto debido a que existen obstáculos para su adopción en aplicaciones específicas quizás por el desconocimiento mismo de las propiedades del material. Se deben realizar cambios en las acciones estratégicas para superar las barreras identificadas. Esta es una importante área de investigación y puede servir como ruta para futuras investigaciones, pues el procesamiento de CV ofrece un gran potencial para aplicaciones industriales, tales como su uso como agente retenedor de residuos radiactivos, pues el análisis XPS reveló que los grupos aluminatos y silicatos presentes en este tipo de cenizas, proporcionan una superficie eléctrica negativa, propiedad muy importante cuando de agentes retenedores se habla. La evaluación económica de las aplicaciones daría una mayor indicación de las áreas en que debe centrarse la investigación, actualmente son escasos los datos económicos de la aplicación de CV en la región y en el país.

\section{Agradecimientos}

Los autores agradecen a la Dirección de Investigaciones de la Universidad Pedagógica y Tecnológica de Colombia, por la financiación de los proyectos SGI-1608 y SGI-1717; a la Compañía Eléctrica de Sochagota S.A. E.S.P - TERMOPAIPA IV, en especial al Ing. WILLIAM CASTELLANOS por suministrar el material para su caracterización, así como por los aportes y comentarios realizados. Al Grupo de Materiales Avanzados de Baja Dimensionalidad, del Instituto de Ciencia de Materiales de Madrid, ICMM-CSIC.

\section{Referencias}

Ahmaruzzaman, M. (2010). A review on the utilization of fly ash. Progress in Energy and Combustion Science, 36, 327-363. doi: https://doi.org/10.1016/j. pecs.2009.11.003

Alhozaimy, A., Soroushian, P., \& Mirza, F. (1996). Effects of curing conditions and age on chloride permeability of Fly Ash mortar. American concrete Institute Materials Journal, 93, 87-95.

American Society for Testing and Materials (ASTM), C618 (2005). Standard specification for coal fly ash and raw or calcined natural pozzolan for use in concrete.

Ampadu, K. O., \& Torii, K. (2002). Chloride ingress and steel corrosion in cement mortars incorporating low quality Fly Ashes. Cement and Concrete Research, 32, (6) 893-901. doi: https://doi. org/10.1016/S0008-8846(02)00721-4

Aperador, W., Ruíz, E., \& Delgado, A. (2014). Electrochemical Impedance Spectroscopy Analysis on Steel Embedded in a Concrete Alkali Exposed on the Chloride Media. International Journal of Electrochemical Science, 9, $7506-7517$.

Baoyi, L., Yuping, D., \& Shunhua, L. (2012). The electromagnetic characteristics of fly ash and absorbing properties of and Building cement-based composites using fly ash as cement replacement. Construction and Build Materials Journal, 27, 184188. doi: https://doi.org/10.1088/2053-1591/ aa7025

Chujiang, C., Shen, Z., Wang, M., ma, S., \& Xing, Y. (2003). Surface metallization of cenospheres and precipitators by electroless plating. China particu- 
ology, 1(4), 156-161. doi: https://doi.org/10.1016/ S1672-2515(07)60133-X

Fauzi, A., Fadhil M. N., B, M. A., \& Al Bakri, A. M. (2016). Study of Fly Ash Characterization as a Cementitious Material. Procedia Engineering 148, 487 - 493. doi: https://doi.org/10.1016/j.proeng.2016.06.535

Fly Ash India Pvt. Ltd. (2014). Recuperado de: flyashindia.com.

Jegadeesan, G., Souhail, R., Abed, A., \& Pinto, P. (2008). Influence of trace metal distribution on its leachability from coal fly ash. Fuel, 87 (1011), 1887-1893. doi: https://doi.org/10.1016/j. fuel.2007.12.007

Goma, F. (1975). El cemento portland y otros aglomerantes, Barcelona España: Editores técnicos asociados s, a.

Gómez-Rojas, O., Díaz-Lagos, M., Blandón-Montes, A., \& Martínez-Ovalle, S. (2016). Presencia de elementos contaminantes como $\mathrm{Cd}, \mathrm{As}, \mathrm{Pb}$, Se y $\mathrm{Hg}$ en carbones de la zona Cundiboyacense, Colombia. Revista de Investigación, Desarrollo e Innovación, 7 (1), 141-150. doi: http://dx.doi. org/10.19053/20278306.v7.n1.2016.5604

Gopalan, M. K. (1996). Sorptivity of fly ash concretes. Cement and Concrete Research, 26 (8), 1189 1197. doi: 10.1016/0008-8846(96)00105-6

Ha, T.H., Muralidharan, S., Bae, J.H., Ha, Y.C., Lee, H.G., Park, K.W., \& Kim, D.K. (2005). Effect of unburnt carbon on the corrosion performance of fly ash cement mortar. Construction and Build Materials Journal, 19 (7) 509-515. doi: https://doi. org/10.1016/j.conbuildmat.2005.01.005

Hemalatha, T., \& Ramaswamy, A. (2017). A review on fly ash characteristics e Towards promoting high volume utilization in developing sustainable concrete. Journal of Cleaner Production 147,
546-559. doi:http://dx.doi.org/10.1016/j.jclepro.2017.01.114

Jensen, A. D., \& Rasmussen, M. S. (2008). A review of the interference of carbon containing fly ash with air entrainment in concrete. Progress In Energy and Combustion Science, 34, 135-154. doi: https:// doi.org/10.1016/j.pecs.2007.03.002

Lieberman, R. N., Green, U., Segev, G., Polat, M., Mastai, Y., \& Cohen, H. (2015). Coal fly ash as a potential fixation reagent for radioactive wastes. Fuel, 153, 437-444. doi: https://doi.org/10.1016/j. fuel.2015.02.111

López, J. C., \& González, C. J. (1995). Manual de reutilización de residuos de la industria minera, siderometalúrgica y termoeléctrica. Instituto Tecnológico Geominero de España, Rivadeneyra, SA.

Lorenzo-García, M. P. (1993). Influencia de dos tipos de cenizas volantes españolas en la microestructura y durabilidad de la pasta de cemento portland hidratado (tesis de doctorado). Universidad complutense de Madrid, Madrid, España.

Martínez-Bernal, M. S. (2013). Determinación de la productividad y competitividad de la pequeña minería del distrito minero del norte de Boyacá. Revista de Investigación, Desarrollo e Innovación, 3 (2), 72-86. doi: 10.19053/20278306.2168

Martínez-Ovalle, S., Reyes-Caballero, F., \& González-Puin, L. X. (2013). Protección radiológica a trabajadores y público en instalaciones que operan radioisótopos industriales. Revista de Investigación, Desarrollo e Innovación, 3 (2), 120-124. doi: 10.19053/20278306.2166

Mehta, P. K. (1989). Pozzolanic and cementitious by products in concrete. Another look [online]. International Concrete Abstracts Portal, 114, 1-44. doi: $10.14359 / 1835$ 
Nassara, R. U. D., Soroushian, P., \& Ghebrabc, T. (2013). Field investigation of high-volume fly ash pavement concrete. Resources Conservation and Recycling, 73, 78-85. doi: https://doi.org/10.1016/j. resconrec.2013.01.006

Naik, T., Singh, S. H., \& Ramme, B. (1998). Mechanical properties and durability of concrete made with blended fly ash. ACI Materials Journal, 95 (4), 454-62.

Nir, L. R., Green, U., Segev, G., Polat, M., Mastai, Y., \& Cohen, H. (2015). Coal fly ash as a potenctial fixation reagent for radioactive wastes. Fuel, 153 (1), 437-444. doi: https://doi.org/10.1016/j. fuel.2015.02.111

Norma Técnica colombiana NTC 3493, (1993). Ingeniería civil y arquitectura. Cenizas volantes y puzolanas naturales, calcinadas o crudas, utilizadas como aditivos minerales en el concreto de cemento pórtland.

Murayama, N., Yamamoto, H., \& Shibatia, J. (2002). Mechanism of zeolite synthesis from coal fly ash by alkali hidrotermal reaction. International Journal of mineral processing, 64, 1-17. doi: https://doi. org/10.1016/S0301-7516(01)00046-1

Pedraza, S. P., Pineda, Y., \& Gutiérrez, O. (2015). Influence of the unburned residues in fly ash additives on the mechanical properties of cement mortars. Procedia Materials Science 9, 496 - 503. doi: https://doi.org/10.1016/j.mspro.2015.05.022

Querol, X., Alastuey, A., Turiel, J. L. F., \& López, S. A., (1995). Synthesis of zeolites by alkaline activation of ferro-aluminous fly ash. Fuel, 74, 1226-123. doi: https://doi.org/10.1016/0016-2361(95)00044-6

Querol, X., Moreno, N., Umaña, J. C., Alastuey, A., Hernández, E., López, S. A., \& Plana, F. (2002). Synthesis of zeolites from coal fly ash: an overview. International Journal Of Coal Geology, 50,
413-423. doi: https://doi.org/10.1016/S01665162(02)00124-6

Raghu, B. K., Eskandari, B. V. H., \& Reddy, B. V. V. (2009). Prediction of compressive strength of SCC and HPC with high volume fly ash using ANN. Construction and Building Materials, 23, 117-128. doi: https://doi.org/10.1016/j.conbuildmat.2008.01.014

Reyes-Caballero, F., Martínez-Ovalle, S. A., \& Moreno-Gutiérrez, M. (2015). Mossbauer characterization of feed coal, ash and fly ash from a termal power plant. Hyperfine Interactions, 232 (1-3), 141-148. doi: 10.1007/s10751-015-1140-1

Reyes-Caballero, F., Fernández-Morales, F., \& Duarte, J. (2016). Panorama energético. revista de investigación, desarrollo e innovación, 7 (1), 151163. doi: http://dx.doi.org/10.19053/20278306. v7.n1.2016.5605

Sahmaran, M. I., Yaman, I. O., \& Tokyay, M. (2009). Transport and mechanical properties of self-consolidating concrete with high volume fly ash. Cement and concrete composites, 31, 99-106. doi: https:// doi.org/10.1016/j.cemconcomp.2008.12.003

Santaella-Valencia, L.E. (2001). Caracterización física química y mineralógica de las cenizas volantes. Ciencia e Ingeniería Neogranadina, 10, 47-62.

Szumiata, T., Brzozka, K., Gorka, B., Gawronski, M., Gzik-Szumiata M., Swietlik R., \& Trojanowska M., (2014). Iron speciation in coal fly ashes chemical and Mossbauer analysis. Hyperfine Interactions, 226, 483-487. DOI 10.1007/s10751-013-0950-2.

Valderrama, C. P., Agredo, J. T., \& de Gutiérrez, R. M. (2011). Características de desempeño de un concreto adicionado con cenizas volantes de alto nivel de inquemados. Ingeniería e Investigación, 31, 39-46. 
Van der Merwe, E. M., Mathebula, C. L., \& Prinsloo, L. C. (2014). Characterization of the surface and physical properties of South African coal fly ash modified by sodium lauryl sulphate (SLS) for applications in PVC composites. Powder Technology, 266, 70-78. Doi: https://doi.org/10.1016/j.powtec.2014.06.008

Vassilev, S. V., \& Vassileva, C. G. (2005). Methods for Characterization of Composition of Fly Ashes from Coal Fired Power Stations: A Critical Overview. Energy Fuels, 19, 1084-1098. DOI: 10.1021/ef049694d

Vasileva, N. G., Anshits, N.N., Sharonova, O.M., Burdin, M.V., \& Anshits, A. G. (2005). Immobilization of cesium and Strontium Radionuclides in Framework Aluminosilicates with the Use of Porous Glass-Ceramic Matrices Based on Coal Fly Ash Cenospheres. Glass Physics and Chemistry., 31, 637647.
Wang, S., \& Wu, H. (2006). Enviromental Benong utilization of fly ash as low cost adsorbents, Journal of Hazardous Materials, 136, 482-501. DOI: 10.1016/j.jhazmat.2006.01.067

Wu, Y., Wang, C., Tan, Y., Jia, L., \& Anthony, E. J. (2011). Characterization of ashes from a $100 \mathrm{kWth}$ pilot-scale circulating fluidized bed with oxy-fuel combustion. Applied Energy, 88, 2940-2948. doi: https://doi.org/10.1016/j.apenergy.2011.03.007

Yua, J., Lib, X., Fleming, D., Meng, Z., Wang, D., \& Tahmasebia, A. (2012). Analysis on Characteristics of Fly Ash from Coal Fired Power Stations Energy. Energy Procedia, 17, 3-9. doi: https://doi.org/10.1016/j.egypro.2012.02.054

Zyryanov, V. V., Petrov, S. A., \& Matvienko, A. A. (2011). Characterization of spinel and magnetospheres of coal fly ashes collected in power plants in the former USSR. Fuel, 90, 486-492. Doi: https:// doi.org/10.1016/j.fuel.2010.10. 